\title{
A Multi-stage Carcinogenesis Model to Investigate Caloric Restriction as a Potential Tool for Post-irradiation Mitigation of Cancer Risk
}

SHORT

COMMUNICATION

\author{
Shusuke Tani, Benjamin John Blyth, Yi Shang, Takamitsu Morioka, Shizuko Kakinuma, Yoshiya Shimada \\ Department of Radiation Effects Research, National Institute of Radiological Sciences, Chiba, Japan
}

\begin{abstract}
The risk of radiation-induced cancer adds to anxiety in low-dose exposed populations. Safe and effective lifestyle changes which can help mitigate excess cancer risk might provide exposed individuals the opportunity to pro-actively reduce their cancer risk, and improve mental health and well-being. Here, we applied a mathematical multi-stage carcinogenesis model to the mouse lifespan data using adult-onset caloric restriction following irradiation in early life. We re-evaluated autopsy records with a veterinary pathologist to determine which tumors were the probable causes of death in order to calculate age-specific mortality. The model revealed that in both irradiated and unirradiated mice, caloric restriction reduced the age-specific mortality of all solid tumors and hepatocellular carcinomas across most of the lifespan, with the mortality rate dependent more on age owing to an increase in the number of predicted rate-limiting steps. Conversely, irradiation did not significantly alter the number of steps, but did increase the overall transition rate between the steps. We show that the extent of the protective effect of caloric restriction is independent of the induction of cancer from radiation exposure, and discuss future avenues of research to explore the utility of caloric restriction as an example of a potential post-irradiation mitigation strategy.
\end{abstract}

(J Cancer Prev 2016;21:115-120)

Key Words: Caloric restriction, Radiation, Prevention, Mathematical model

\section{INTRODUCTION}

Following exposure of a population to radiation, concern about the risk of radiation-induced cancer can be a major source of anxiety, ${ }^{1,2}$ particularly for parents concerned about the future health of their children. ${ }^{3}$ Despite the obvious importance of preventing accidental radiation exposure and reducing exposure levels, it is not currently known whether there is a practical way to specifically mitigate radiation-induced cancer risk once exposure has occurred. ${ }^{4.5}$ Post-exposure measures to increase survival from acute radiation are available for individuals needing intensive care after high-dose exposure; however, for large populations exposed to low radiation doses, there are no medical interventions which are currently advised to reduce the probability of a radiation-induced malignancy later in life.
Research into chemical and biologic radio-protectors has been ongoing for many decades, ${ }^{6}$ and while some agents have been found to confer a certain degree of protection against acute effects when delivered within a short period after irradiation, many need to be administered prior to exposure in order to be effective. In the absence of a specific medical countermeasure, a consensus regarding other strategies proven to be effective in minimizing radiation-induced cancer risk and/or cancer risk more generally, would likely be a valuable public health tool. In addition to direct effects on cancer burden, empowering individuals in exposed populations by providing safe, proven methods to lower their cancer risk could assist in decreasing anxiety and improving coping skills. ${ }^{7}$

One candidate for such an approach is caloric restriction (CR). Controlled dietary intake has long been studied in terms of its

Received April 19, 2016, Revised May 26, 2016, Accepted May 30, 2016

Correspondence to: Shizuko Kakinuma

Department of Radiation Effects Research, National Institute of Radiological Sciences, 4-9-1 Anagawa, Inage, Chiba 263-8555, Japan

Tel: +81-432063160, Fax: +81-432064138, E-mail: kakinuma.shizuko@qst.go.jp

Copyright (C) 2016 Korean Society of Cancer Prevention

(c) This is an Open Access article distributed under the terms of the Creative Commons Attribution Non-Commercial License (http://creativecommons.org/licenses/by-nc/4.0) which permits unrestricted non-commercial use, distribution, and reproduction in any medium, provided the original work is properly cited. 
effects on increasing longevity and reducing cancer incidence. ${ }^{8}$ CR has also been investigated for its potential as an adjuvant cancer treatment, to slow the growth of existing tumors. ${ }^{9} 10$ Although many specific mechanisms of $\mathrm{CR}$ have been documented, it has effects on a wide range of physiological and cellular systems, not all of which are understood. In fact, the levels of CR required to produce the greatest longevity effects in experimental animals are perhaps beyond what could be reasonably maintained by most people, and certainly would not be advised for children or young people during their developmental years. ${ }^{11}$ Yet, understanding the kinetics of how cancer preventative/suppressive approaches can be instigated long after the time of irradiation affects the risk of radiation-induced cancer can help us to determine the utility of such approaches, and whether they can specifically prevent or merely offset radiation-induced cancer risk.

We recently published the results from a relevant lifespan study in mice. Seven weeks after 1-week-old mice were subjected to $3.8 \mathrm{~Gy}$ irradiation, they were fed either a diet equivalent to their ad libitum calorie intake ( $95 \mathrm{kcal} / \mathrm{mouse} /$ week) or switched to a nutritionally-balanced diet limited to approximately $1 / 3$ fewer calories $\left(65 \mathrm{kcal} / \mathrm{mouse} /\right.$ week). ${ }^{12}$ The results showed that irradiation alone decreased tumor-free lifespan, $\mathrm{CR}$ alone increased tumor-free lifespan, and initiating $\mathrm{CR}$ after irradiation was able to partially mitigate radiation-induced cancer. Separating the data by cause of death revealed that different tumor types/sites responded differently to $\mathrm{CR}$, with radiation-induced lymphomas showing little to no response, while a clear effect could be seen for late-occurring solid tumors. Here, we analysed the pathology results further to identify cases of lethal solid tumors and applied a multistage mathematical model of carcinogenesis to the data ${ }^{13}$ in order to gain mechanistic insight into the protective effect of $\mathrm{CR}$, specifically as it relates to radiation-induced cancers.

\section{MATERIALS AND METHODS}

We used lifespan data from male B6C3F1 mice published by Shang et al. ${ }^{12}$ Briefly, mice were either irradiated with $3.8 \mathrm{~Gy}$ of $\mathrm{X}$-rays at 1 week of age, or were sham-irradiated. This radiation dose was chosen because it is known to efficiently induce both haematopoietic and various solid tumors in the $\mathrm{B} 6 \mathrm{C} 3 \mathrm{~F} 1$ mouse strain, providing the opportunity to examine whether $\mathrm{CR}$ would have differential effects by tumor type. At 7 weeks of age, irradiated and unirradiated mice were switched from an ad libitum diet to either a diet limited to $95 \mathrm{kcal} / \mathrm{week} /$ mouse (equivalent to ad libitum) or to a nutrient-balanced but calorically restricted diet of $65 \mathrm{kcal} /$ week/mouse (a caloric reduction of approximately one-third). At this age, mice are fully developed adults but have not yet reached their peak body weight, allowing the mice to physiologically adapt to long-term $\mathrm{CR}$ without interrupting normal development or inducing a sharp decline in body weight. The four groups were monitored over their natural lifespan, with detailed autopsy and pathology analysis for each mouse. tumor spectrum and latency, and their effects on overall lifespan, were used as measures of the interaction between radiation-induced carcinogenesis and $\mathrm{CR}$.

In the original study, lifespans were compared based on tumors that the mice harboured at the time of autopsy. However, as such tumors could be either incidental or lethal, the age of the mouse at the time of death may not have been directly related to any one of the tumors discovered at autopsy. ${ }^{14}$ Thus, we elected to use data only from mice with tumor(s) that were diagnosed as lethal, with the autopsy and pathology records re-evaluated by a veterinary pathologist. This approach allowed us to compare the age-specific tumor mortality in a manner analogous to that used in human cancer epidemiology. Since $\mathrm{CR}$ was not observed to have a significant effect on leukaemia/lymphoma, we limited our analyses to deaths due to any solid tumor (except sarcoma, which was rare), and then further analysed deaths due to lethal hepatocellular carcinoma (HCC), lethal lung tumor, or lethal hemangioma.

We used a simple implementation of the Armitage-Doll model, ${ }^{13}$ which postulates that a normal single cell must undergo several critical steps, such as mutations or other rate-limiting events, to become a malignant cell. Armitage and Doll used this model to explain the temporal variation in the death rate for solid cancer, and it has since been used across a large number of epidemiological studies to estimate the stages of cancers at many sites. ${ }^{15.16}$ The mortality rate of cancer at age $t, \mathrm{I}(t)$ (tumor deaths/mouse-day), is related to the probabilities of the transition between each step, $p$ (transitions per unit time). Because the transitions must proceed in a unique, sequential order, $\mathrm{I}(t)$ is expressed as shown in Equation (1), below, where $k$ is the number of critical stages in the course of carcinogenesis a cell needs to pass through before becoming fully malignant, $t$ is the age of the mice, and $p_{1}$ is the probability of a transition from the $(i-1)^{\text {th }}$ to the $i^{\text {th }}$ change $(i \leq k)$.

$$
\text { (1) } \mathrm{I}(t)=\frac{p_{1} p_{2} \cdots p k}{(k-1) !} t^{k-1}
$$

The logarithm of $\mathrm{I}(t)$ is directly proportional to the logarithm of age, as shown in Equation (2), where a is a constant representing 
the product of the transition $\left(\mathrm{P}_{\mathrm{k}}\right)$ rates divided by $(k-1)$ !, and $t$ (in our analysis) is the mid-point of age divided into 200-day increments.

$$
\text { (2) } \log \mathrm{I}(t)=\mathrm{a}+(k-1) \log t
$$

By plotting the log of age-specific mortality rate, $\mathrm{I}(t)$, against the log of age $(t)$ obtained from the lifespan and pathology data, we used a linear fit (estimated using the non-linear least squares method with $\mathrm{R}$ software version 3.1.3) to calculate the intercept (a) and slope ( $k-1)$ for each of the three site-specific tumors and all solid tumors to derive the model parameters $\mathrm{P}_{\mathrm{k}}$ and $k$. An estimate of the per-stage transition rate, $p_{k}$, was calculated from

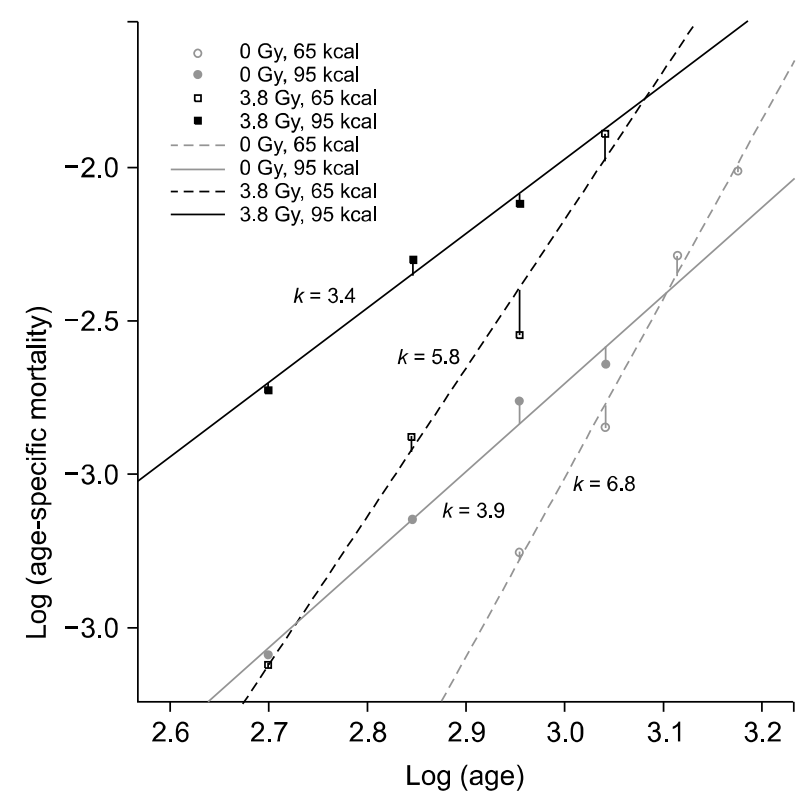

Figure 1. Armitage-Doll model fits of age-specific mortality data taken from Shang et al., ${ }^{12}$ calculated from deaths from all solid cancers (except sarcoma). The data points are plotted as the log of age-specific mortality (tumor-deaths per mouse day) versus the log of age (days) for each incremental 200-day period. The lines show the fit used to calculate the model parameters $k$ and $a$, and the $k$ parameter derived from each fit is shown. Drop lines from each point show the deviations from the fit. the product of the transition rates, $\mathrm{P}_{\mathrm{k}}$.

\section{RESULTS}

Figure 1 shows the model fit for all solid tumors for the four treatment groups, with the model parameters for all solid tumor data and for the three site-specific analyses shown in Table 1. The data clearly show a more rapid increase in the mortality rate with age in the two $65 \mathrm{kcal}$ groups (CR) compared to the mortality rate of mice on the $95 \mathrm{kcal}$ diet. This is consistent with the increased lifespan associated with CR reported in the original data, since, although there is a stronger effect of age on the increase in the mortality rate, the mortality rate starts at a lower point and remains lower for most of the animals' lifespans, exceeding that of the standard diet only at the most advanced ages, by which time few animals remain alive. The increase in the slope and thus in the model parameter $k$ with $\mathrm{CR}$ is significant for all solid tumors, HCC, and lung tumor deaths (Table 2), but is not significant for hemangioma. For all solid tumors and HCC, the increase in $k$ associated with $\mathrm{CR}$ was equivalent in irradiated and unirradiated mice. On the contrary, irradiation did not change the estimate of $k$, with no significant change in the slopes of the graphs of solid tumor deaths or any of the site-specific data.

For each of the four treatment groups, the age-specific mortality data for all solid tumors shows an increase with age that is consistent with the power function characteristic of the simple Armitage-Doll model ( $\left.\mathrm{R}^{2}>0.98\right)$. HCC represented between $27 \%$ to $56 \%$ of the diagnoses for solid tumor death in each group, and the model for the HCC data $\left(\mathrm{R}^{2}>0.93\right)$ was very consistent with the all-solid tumor model. Although the model fits were reasonable $\left(\mathrm{R}^{2}>0.88\right)$, there were too few cases of lung cancer to provide a model fit for each group, and the model fit was poor for hemangioma $\left(\mathrm{R}^{2}>0.38\right)$.

\section{DISCUSSION}

Both the pooled solid tumor and HCC models showed a

Table 1. Parameters of the Armitage-Doll model for deaths from all solid cancers and site-specific tumors

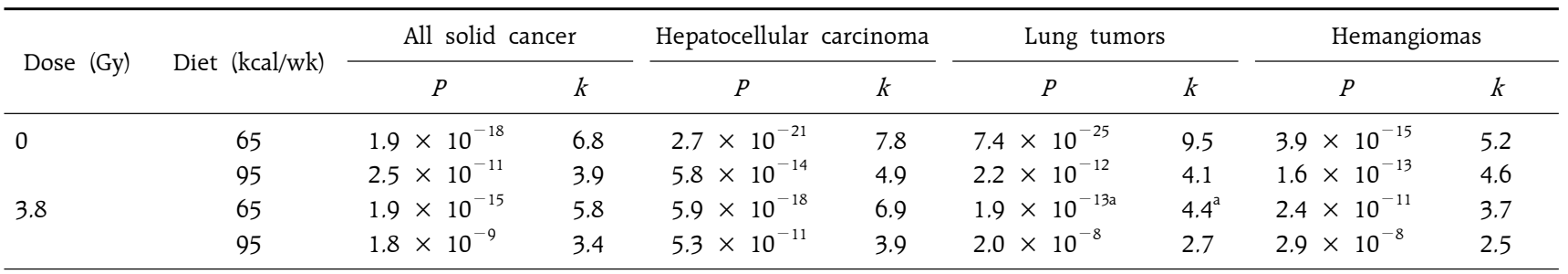

${ }^{\mathrm{a}}$ This value is for reference only, based on a fit to only two points from the experimental data. 
Table 2. Changes of the stages $(\triangle K)$ affected by calorie and irradiation

\begin{tabular}{|c|c|c|c|c|c|c|}
\hline & \multicolumn{3}{|c|}{ Effect of $\mathrm{CR}(\Delta k \pm \mathrm{SE})$} & \multicolumn{3}{|c|}{ Effect of Irradiation $(\triangle k \pm \mathrm{SE})$} \\
\hline & 0 Gy & $3.8 \mathrm{~Gy}$ & Difference with radiation & $65 \mathrm{kcal}$ & $95 \mathrm{kcal}$ & Difference with $C R$ \\
\hline All solid tumors & $2.9 \pm 0.5^{*}$ & $2.4 \pm 0.6^{*}$ & -0.5 & $-1.0 \pm 0.7$ & $-0.5 \pm 0.4$ & -0.5 \\
\hline $\mathrm{HCC}$ & $2.9 \pm 1.0 *$ & $3.0 \pm 1.0$ & 0.1 & $-0.9 \pm 0.8$ & $-1.0 \pm 1.2$ & 0.1 \\
\hline Lung cancer $^{a}$ & $5.4 \pm 1.9 *$ & $1.7^{\mathrm{a}}$ & - & $-5.1^{\mathrm{a}}$ & $-1.4 \pm 0.9$ & . \\
\hline Hemangioma & $0.6 \pm 1.2$ & $1.2 \pm 1.9$ & 0.6 & $-1.5 \pm 1.0$ & $-2.1 \pm 2.3$ & 0.4 \\
\hline
\end{tabular}

$\mathrm{CR}$, caloric restriction; HCC, hepatocellular carcinoma. ${ }^{a}$ These values are derived from a slope co-efficient based on only two age-specific mortality points in the $3.8 \mathrm{~Gy}-65 \mathrm{kcal}$ group. ${ }^{*}$ The difference in slopes is statistically significant $(P<0.05)$.

significant increase in $k$ with CR. An increase in $k$ of around 3 reflects a more rapid increase in the mortality rate in aging mice on the lower calorie diet, albeit starting from a lower baseline mortality rate. According to the tenets of the model, these data could be construed as reflecting the presence of additional 'steps' necessary for the carcinogenic process which delay the onset of tumors but also allow for the accumulation of cells in the penultimate stages of tumor formation. The reduction in the product of the transition rates with $\mathrm{CR}$ could be the result of an overarching effect common to all of the steps (such as a decrease in the spontaneous mutation rate), or the result of a specific effect which alters the probability of one particular transition. The results presented here confirm that solid tumor mortality is indeed lower when mice are subjected to CR for most of their lifespan, and excludes artefacts due to variations in tumor stage or accelerated discovery of incidental tumors due to earlier death caused by another tumor type.

The similarity in the effect of $\mathrm{CR}$ on both parameters between the irradiated and unirradiated groups may suggest that there is no or little interaction between the detrimental effects of irradiation and the beneficial effects of the dietary regimen. It is thus possible that CR offsets both spontaneous and radiation-induced carcinogenesis in a similar fashion. This could mean that existing data on the optimal levels, duration, and timing of CR may be applicable to individuals who have previously been exposed to radiation. Interestingly, although the products of the transition rates did increase, the modelled values of $k$ were not significantly lower in the irradiated mice, consistent with the notion that the probability of mutations was altered without concomitant alterations in the oncogenic pathway. It may be that a radiation-induced DNA mutation, such as inactivation of a tumor suppressor gene, might instantaneously move cells one or more steps forward through a multistage progression towards carcinogenesis, effectively removing the step(s). Although not significant, the effect of irradiation on the values of $k$ were estimated to be in the range of a loss of a single step, and, thus, such an explanation is not excluded by our data. Since radiation may act on the carcinogenic process by inducing genomic instability, stimulating tissue regeneration following radiation-induced apoptosis, and other physiological changes, as well as introducing DNA mutations, it is plausible that the overall effect of irradiation on age-specific mortality would affect both the number of steps and the transition rate.

Here, the effects of radiation and $\mathrm{CR}$ were not included in the model itself, since a sufficient quantity of data are not currently available to formalize the relationship. However, having demonstrated an effect of $\mathrm{CR}$ on both the parameters of this simple model, we can begin to consider the types of experimental data that would be valuable for incorporating radiation exposure and CR directly into the model. The most pertinent questions pertaining to $\mathrm{CR}$ would be:

- What is the relationship between the level of $\mathrm{CR}$ and the induction of protective effects, and is there a threshold?

- What is the effect of the interval between radiation exposure and the onset of $\mathrm{CR}$ ?

- What is the effect of the duration of CR?

In one study, adult $\operatorname{Tr} p 53^{+/-}$mice (which are highly predisposed to cancer development) were assigned to either a calorie-restricted diet or a one-day-a-week fasting regimen, and both diets were associated with a delay in cancer-related death. ${ }^{17}$ As discussed above, if radiation-mediated cancer induction and CR-mediated cancer suppression are indeed independent of one another, existing data on $\mathrm{CR}$ regimens could be used to formulate a model to examine optimal strategies for offsetting both radiation-induced and spontaneous cancer risk. Such approaches have been used to model lung cancer risk after smoking cessation to understand the competing effects of smoking levels, duration, and time since quitting. ${ }^{18}$ Analogously, comparing the effectiveness of long-term/lifetime $C R$ versus short-term intense $C R$ after irradiation might provide insight into the nature of the additional steps implied by the model discussed here, and such animal experiments are now being undertaken. 
Ultimately, the impractical nature of extreme $\mathrm{CR}$ gives rise to the question of whether complementary stressors or mimetics could be used instead. Much research has been undertaken to find CR mimetics, ${ }^{19}$ and candidates which prove effective will likely be applicable to post-radiation risk mitigation. We are investigating the effect of addition of dietary supplements (such as the plant phenol resveratrol, one candidate $\mathrm{CR}$ mimetic ${ }^{20}$ ) to the standard laboratory diet of mice. We are also undertaking experiments to determine whether radiation-induced tumors arising in calorie-restricted and -unrestricted animals show any differences in key carcinogenic pathways at the molecular level. Any differences in tumor phenotype may indicate pathways where $\mathrm{CR}$ interacts with the accumulation or selection of mutations. In parallel, we have established experiments utilizing environmental enrichment for laboratory mice (play equipment, increased housing space, nesting materials) following radiation exposure to explore whether other simple lifestyle changes may mitigate radiation-induced cancer. It is not expected that any one of these measures alone will have a dramatic impact on cancer incidence, but lifestyle changes that are safe, simple, and beneficial represent low-hanging fruit that might be included in post-disaster counselling and public health campaigns. Human populations invariably have additional cancer risk factors, ${ }^{21}$ such as smoking, alcohol consumption, sedentary lifestyles, and associated obesity, which are difficult to reproduce in experimental animals. However, it is likely that existing public health recommendations for reducing cancer risk will be similarly effective in offsetting or mitigating low dose radiation-induced cancer risk.

Ultimately, at the radiation exposure levels relevant to large populations in the wake of environmental contamination, the increased risk to the individual is small compared to the background risk of cancer in the population. Simple lifestyle changes which can be proven to help offset both spontaneous and radiation-induced risk might provide significant public health benefits, both in terms of decreased cancer burden and improved mental health and well-being.

\section{ACKNOWLEDGMENTS}

We would like to thank the support staff of the Department of Radiation Effects Research for their assistance with providing access to the autopsy and pathology data.

\section{CONFLICTS OF INTEREST}

No potential conflicts of interest were disclosed.

\section{REFERENCES}

1. Sugimoto T, Shinozaki T, Naruse T, Miyamoto Y. Who was concerned about radiation, food safety, and natural disasters after the great East Japan earthquake and Fukushima catastrophe? A nationwide cross-sectional survey in 2012. PLoS One 2014;9:e106377.

2. Goto A, Reich MR, Suzuki Y, Tsutomi H, Watanabe E, Yasumura S. Parenting in Fukushima city in the post-disaster period: short-term strategies and long-term perspectives. Disasters 2014;38 Suppl 2:S179-89.

3. Bromet EJ. Emotional consequences of nuclear power plant disasters. Health Phys 2014;106:206-10.

4. Stone HB, Moulder JE, Coleman CN, Ang KK, Anscher MS, Barcellos-Hoff MH, et al. Models for evaluating agents intended for the prophylaxis, mitigation and treatment of radiation injuries. Report of an NCI Workshop, December 3-4, 2003. Radiat Res 2004;162:711-28.

5. Movsas B, Vikram B, Hauer-Jensen M, Moulder JE, Basch E, Brown SL, et al. Decreasing the adverse effects of cancer therapy: national cancer institute guidance for the clinical development of radiation injury mitigators. Clin Cancer Res 2011;17:222-8.

6. Singh VK, Ducey EJ, Brown DS, Whitnall MH. A review of radiation countermeasure work ongoing at the armed forces radiobiology research institute. Int J Radiat Biol 2012;88:296-310.

7. Oughton D. Societal and ethical aspects of radiation risk perception. In: eds, by Shigemura J, Chhem R, Mental Health and social Issues Following a Nuclear Accident: The Case of Fukushima. Tokyo, Springer, pp 45-54, 2016.

8. Hursting SD, Smith SM, Lashinger LM, Harvey AE, Perkins SN. Calories and carcinogenesis: lessons learned from 30 years of calorie restriction research. Carcinogenesis 2010;31:83-9.

9. Klement RJ, Champ CE. Calories, carbohydrates, and cancer therapy with radiation: exploiting the five R's through dietary manipulation. Cancer Metastasis Rev 2014;33:217-29.

10. Morscher RJ, Aminzadeh-Gohari S, Feichtinger RG, Mayr JA, Lang $\mathrm{R}$, Neureiter $\mathrm{D}$, et al. Inhibition of neuroblastoma tumor growth by ketogenic diet and/or calorie restriction in a CD1-Nu mouse model. PLoS One 2015;10:e0129802.

11. Cava E, Fontana L. Will calorie restriction work in humans? Aging (Albany NY) 2013;5:507-14.

12. Shang Y, Kakinuma S, Yamauchi K, Morioka T, Kokubo T, Tani S, et al. Cancer prevention by adult-onset calorie restriction after infant exposure to ionizing radiation in $\mathrm{B} 6 \mathrm{C} 3 \mathrm{~F} 1$ male mice. Int $\mathrm{J}$ Cancer 2014;135:1038-47.

13. Armitage P, Doll R. The age distribution of cancer and a multi-stage theory of carcinogenesis. Br J Cancer 1954:8:1-12.

14. Heidenreich WF, Collier C, Morlier JP, Cross FT, Kaiser JC, Monchaux G. Age-adjustment in experimental animal data and its application to lung cancer in radon-exposed rats. Radiat Environ Biophys 2004:43:183-8.

15. Pierce DA, Vaeth M. Age-time patterns of cancer to be anticipated from exposure to general mutagens. Biostatistics 2003:4:231-48.

16. Frank SA. Dynamics of cancer: incidence, inheritance, and evolu- 
tion (2010/09/08 ed.). Princeton, Princeton University Press, 2007.

17. Berrigan D, Perkins SN, Haines DC, Hursting SD. Adult-onset calorie restriction and fasting delay spontaneous tumorigenesis in p53-deficient mice. Carcinogenesis 2002;23:817-22.

18. Brown $\mathrm{CC}, \mathrm{Chu} \mathrm{KC}$. Use of multistage models to infer stage affected by carcinogenic exposure: example of lung cancer and cigarette smoking. J Chronic Dis 1987;40 Suppl 2:171S-9S.

19. Lane MA, Roth GS, Ingram DK. Caloric restriction mimetics. In: ed, by Tollefsbol TO, Biological Aging: Methods and Protocols; Methods in Molecular Biology. New York, Humana Press, pp 143-9, 2007

20. Bishayee A. Cancer prevention and treatment with resveratrol: from rodent studies to clinical trials. Cancer Prev Res (Phila) 2009:2:409-18.

21. Anand P, Kunnumakkara AB, Sundaram C, Harikumar KB, Tharakan ST, Lai OS, et al. Cancer is a preventable disease that requires major lifestyle changes. Pharm Res 2008:25:2097-116. 